\title{
A List of Stars in the $\beta$ Cephei and SPB Instability Strips
}

\author{
P. Blay, J. Fabregat \\ University of Valencia, Instituto de Ciencias de los Materiales, \\ Departamento de Astronomía y Astrofísica, Avda. Dr. Moliner 50, \\ 46100 Burjassot, Spain
}

For the purpose of observing $\beta$ Cephei and SPB stars with optical telescopes on board future space missions, we have developed an automatic photometric search of stars falling inside the $\beta$ Cephei and SPB instability strips. A list has been compiled which is available upon request from the authors (blay@castor.daa.uv.es; juan@pleione.daa.uv.es).

The boundaries of the instability strips have been defined by observational $\beta-c_{0}$ HR diagrams based on Strömgren photometry (Sterken \& Jerzykiewicz $1993=$ SK93). The ZAMS has been set as the lower limit of both strips. Low and high temperature limits are set as those given by SK93 and the upper limit is fixed by the photometry $\beta$ of Cep stars found in the literature. For the SPB stars we have followed the same procedure. We have found the following boundaries: $\beta$ Cephei: $0.2295 c_{0}+2.6263>\beta>0.0394 c_{0}+2.5656 ; 0.17>c_{0}>-0.1$; SPB: $2.630>\beta>0.2642 c_{0}+2.6207 ; 0.8>c_{0}>0.17$.

In the case of Johnson $U B V$ photometry the boundaries for the instability strips have been set taking into account just the $Q$ index. It has been calculated for almost the same set of stars as above. The boundaries that we have found are the following: $\beta$ Cephei: $-0.92<Q<-0.64$; SPB: $-0.64<Q<-0.28$.

Using the boundaries described above we have made a photometric selection of stars in the Hauck \& Mermilliod (1998) $u v b y-\beta$ and Mermilliod $(1987,1994)$ $U B V$ catalogs. We have collected in a first list a total of 8143 stars. As an example of the suitability of our lists as a part of the observing program for optical space telescopes, we have compared it with the performances of the INTEGRAL Optical Monitoring Camera (OMC, Giménez et al. 1998). The best magnitude interval in which the OMC be able to observe ranges from 10 to 19. A photometric accuracy better than $0.01 \mathrm{mag}$ will be reached. More than a half of the stars of our sample, namely 4568 , fall in this magnitude range, and hence are suitable for the study of pulsational variations with the OMC.

\section{References}

Giménez, A., Mas-Hesse, J.-M., Jamar, C., et al. 1998, in Proc. 3rd INTEGRAL Workshop, The Extreme Universe, ESA-SP (in press)

Hauck, B. \& Mermilliod, M. 1998, A\&AS, 129, 431

Mermilliod, J.-C. 1987, A\&AS, 71, 413

Mermilliod, J.-C. 1994, Bull. Inf. Centre Donées Stellaires, 45, 3

Sterken, C. \& Jerzykiewicz, M. 1993, Space Sci. Rev., 62, 95 